\title{
Detecting Episodes of Brady- and Tachycardia Using Photo-plethysmography at the Wrist in Free-living Conditions
}

\author{
Alberto G Bonomi ${ }^{1}$, Linda M Eerikäinen ${ }^{1,2}$, Fons Schipper ${ }^{1}$, Ronald M Aarts ${ }^{1,2}$, Helma M de \\ Morree $^{1}$, Lukas Dekker ${ }^{2,3}$ \\ ${ }^{1}$ Personal Health Department, Philips Research, Eindhoven, the Netherlands \\ ${ }^{2}$ Department of Electrical Engineering, Eindhoven University of Technology, the Netherlands \\ ${ }^{3}$ Department of Cardiology, Catharina Hospital, Eindhoven, the Netherlands
}

\begin{abstract}
Detecting episodes of bradycardia and tachycardia can help identifying the clinical relevance of common cardiac symptoms. This study aimed at investigating whether an unobtrusive wrist-wearable device equipped with a photoplethysmographic (PPG) and acceleration sensor could be used to detect such rate abnormalities in free-living conditions. Twenty patients $(M=55 \%$, age: $67 \pm 13 y)$ reporting cardiac symptoms were monitored for 24 hours in free-living conditions using a portable Holter ECG recorder. Simultaneously, a wrist-wearable device equipped with a PPG and acceleration sensor was used to measure heart rate and the mean inter-pulse-interval (IPI) in 5-sec epochs. ECG-derived inter-beat-intervals (IBI) were used as ground truth for determining episodes of bradycardia $(>1200 \mathrm{~ms})$ and tachycardia $(<500 \mathrm{~ms})$ during the monitoring period. According to the ECG, the duration of brady-and tachycardia and normal rate lasted a total of $766 \mathrm{~min}, 64 \mathrm{~min}$, and $27024 \mathrm{~min}$, respectively. Average IPI during bradycardia and tachycardia was $1310 \pm 80 \mathrm{~ms}$ and $459 \pm 37 \mathrm{~ms}$, respectively. IPI data correctly identified episodes of bradycardia ( $\mathrm{Se}: 85.0 \%$, Sp: $99.4 \%)$ and tachycardia (Se: 89.1\%, Sp: 99.9\%). In conclusion, a wrist-wearable device equipped with a PPG sensor can accurately detect rate abnormalities such as brady-and tachycardia in free-living conditions.
\end{abstract}

\section{Introduction}

Heart rate abnormalities such as bradycardia and tachycardia are defined as excessively suppressed or elevated rates of the heart, respectively. Such conditions are usually well tolerated in healthy subjects and can occur in response to specific situations such as sleep, relaxation or physical activity. However, bradycardia and tachycardia often appear in presence of cardiac pathologies and patients may suffer from intermittent and subtle symptoms [1]. Bradycardia is defined as a heart rate (HR) lower than
$60 \mathrm{bpm}$, but generally a HR $<50 \mathrm{bpm}$ is needed to cause significant symptoms such as fatigue, exercise intolerance, and transient dizziness. Tachycardia presents itself with HR higher than $120 \mathrm{bpm}$ and can lead to palpitations, shortness of breath or dyspnea on exertion [2]. Onset of tachycardia could even represent a medical emergency in presence of other cardiac pathologies such as underlying coronary artery disease or ventricular dysfunction [3].

Long-term and continuous monitoring of bradycardia and tachycardia is beneficial to evaluate initiating factors such as stress, medications, physical activity or sleep as well as the interrelation with patient-reported symptoms. The most commonly used methods to investigate the presence of HR abnormalities are 12-lead ECG, 24-hour Holter monitor or 30-day event recorder if the symptoms do not occur daily [4]. Yet, an unobtrusive solution for long-term monitoring purposes of bradycardia and tachycardia is not available.

In recent years, wrist-wearable photo-plethysmographic (PPG) sensors have been developed to continuously and unobtrusively measure HR in free-living conditions [5]. We previously showed that the same technology could be used to accurately detect heart rhythm irregularities due to atrial fibrillation [6]. Therefore, such wrist-wearable sensor could provide rich information to a physician on the presence and pattern of rate and rhythm abnormalities in symptomatic patients. However, the accuracy of PPGbased methods used to determine HR has never been tested at the limits of the physiological range.

In this study we sought to investigate whether PPG and acceleration data at the wrist could detect episodes of HR abnormalities in free-living conditions. Additionally we investigated the effect of movement artefacts on the detection accuracy of brady- and tachycardia as compared to adjudications based on ECG recordings.

\section{Methods}

We conducted an observational study aimed at comparing the outcome of a clinical test based on an ECG 
Holter monitor to an algorithm based on PPG data to detect episodes of HR abnormalities such as bradycardia and tachycardia in free-living conditions (Figure 1).

\subsection{Participants and protocol}

Volunteers were male and female patients scheduled for a 24-hour Holter ECG test. All the volunteers provided written informed consent. The institutional medical ethical committee approved the study.

Volunteers reported in the morning to the cardiology department of the hospital for a routine installation of a Holter monitor. Subsequently, a wrist-wearable device equipped with a PPG and acceleration sensor was positioned on the wrist of the non-dominant arm for the entire duration of the Holter monitoring period. Volunteers were then free to go home and carry out their normal routine. The protocol ended after 24 hours when the Holter test ended and the patient returned to the hospital. A synchronization procedure consisting of pressing the event marker on the Holter while tapping on the case of the wristwearable device was carried out twice (at the beginning and end of the monitoring period) to allow time alignment of the collected data. Patient information was obtained from the medical records.

\subsection{Wrist-wearable sensing device}

The PPG signal and body acceleration were measured using a wrist-wearable sensor equipped with the Philips Cardio and Motion Monitoring Module (CM3 Generation3 , Philips), which is an integrated module including optical PPG and accelerometer sensors. The PPG sensor is based on a reflective modality and the light source is formed by two green light LEDs. Sampling frequency of the raw data was set to $128 \mathrm{~Hz}$ and the dynamic range of the accelerometer was $\pm 8 \mathrm{~g}$. The raw PPG and accelerometer signal was processed to define the average HR at intervals of 1 sec. Validation of those HR measurements was provided in previous publications [7-9]. The estimated HR was used to determine the average inter-pulse interval (IPI) indicating the mean time between pulses related to blood volume changes underneath the skin. We used this feature as a surrogate value for inter-beat intervals (typically extracted from ECG data). The accelerometer signal was processed to determine movement intensity on a sec-bysec basis. The equation below defined movement intensity:

$$
\text { Movement Intensity }=\sum_{a x=1}^{3}\left[\sum_{i=1}^{N}\left(a c c_{a x}^{i}-m_{a x}\right)^{2}\right]
$$

where $a c c_{a x}$ is the acceleration signal for each sensing axis of the accelerometer $(a x)$, and $m_{a x}$ the mean acceleration of $N$ acceleration samples.

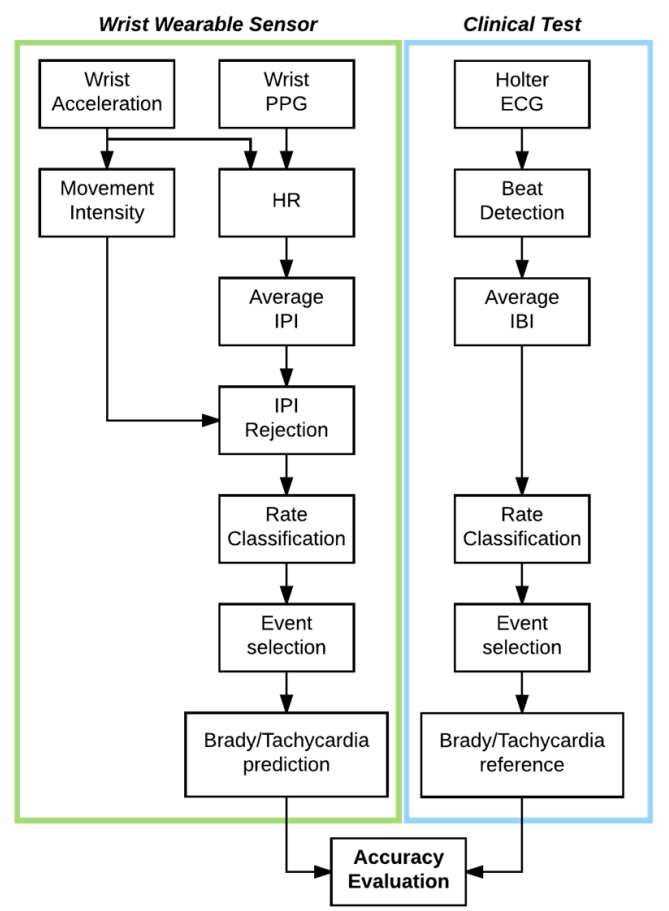

Figure 1. Processing flow to detect bradycardia and tachycardia from wrist PPG and acceleration data and evaluate detection accuracy.

\subsection{Holter ECG measurements}

The ECG of the patients was measured using a continuous 12-lead Holter recorder (H12+, Mortara, Milwaukee, WI, USA). This device provided as output the time of each detected heartbeat (Veritas, Mortara, Milwaukee, WI, USA) which was used to determine the inter-beat interval (IBI) and thereby the ground truth for events of brady- and tachycardia. According to clinical practice, bradycardia was defined as any episode of length longer than 3 minutes during which the IBI was $>1200 \mathrm{~ms}$ $(\mathrm{HR}<50 \mathrm{bpm})$. Similarly tachycardia was defined as any episode of duration longer than 3 minutes during which the IBI did not exceed $500 \mathrm{~ms}$ (HR $>120 \mathrm{bpm}$ ). Those episodes where considered as the ground truth to use as target for the evaluation of the PPG-based HR abnormalities detection method.

\subsection{HR abnormalities detection from PPG}

The IPIs were calculated in 5-sec windows from PPGderived estimates of HR. Such IPIs were then used to identify periods of bradycardia and tachycardia. Analysis periods were removed when the movement intensity exceeded a threshold indicating that the patient was physically active to remove movement artefacts. Clinical rules for defining brady- and tachycardia were applied to IPI data. Ultimately an event selection process was used to 
only retain those series of brady- or tachy-IPIs that lasted more than 3 minutes (Figure 1). The outcome of such analysis was a series of events labelled as bradycardia, tachycardia, or normal heart rate from PPG data.

\subsection{Statistics}

The outcome of the IPI-based rate classification algorithm was compared to the output of the Holter ECG reference for each patient. The two datasets were synchronized by time aligning the two markers in the Holter with the acceleration signal resulting from the tapping events on the wrist recorder. Further confirmation of the synchronization parameters (time offset and gain) was determined by maximizing the correlation between the IBI and IPI series. The accuracy of the bradycardia, tachycardia, and normal rate classification algorithm was defined by its sensitivity and specificity according to the confusion matrix.

\section{Results}

Volunteers were 20 patients (males: $55 \%$, age: $67.4 \pm$ 13.0 y, BMI: $28.1 \pm 5.3 \mathrm{~kg} / \mathrm{m}^{2}$ ). Rate- and rhythm-control medication was prescribed to $50 \%$ and $40 \%$ of the participants, respectively. About 460 hours of ECG recordings were collected simultaneously with PPG and wrist acceleration data in free-living conditions. According to the Holter ECG reference none of the patients suffered from atrial fibrillation or any other rhythm disorder but many premature beats were identified. A strong correlation was found between IBI and IPI data once averaged on a minute-by-minute basis $\left(\mathrm{R}^{2}=81 \pm 20 \%\right)$. According to ECG data, episodes of tachycardia were documented in only 2 patients. The total duration of episodes of tachycardia lasted 64 minutes. On the other hand, episodes of bradycardia were found in 5 patients for a total duration of 766 minutes.

Table 1. Confusion matrix for HR abnormalities detection

\begin{tabular}{|c|c|c|c|c|c|c|}
\hline \multirow{8}{*}{ 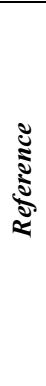 } & \multicolumn{4}{|c|}{ Predicted } & \multirow{3}{*}{$\begin{array}{c}\text { Se } \\
85.0 \%\end{array}$} & \multirow{3}{*}{$\begin{array}{c}\text { Sp } \\
99.4 \%\end{array}$} \\
\hline & & Brady & Normal & Tachy & & \\
\hline & Brady & 7455 & 1312 & 0 & & \\
\hline & Normal & 1317 & 235409 & 142 & $99.4 \%$ & $85.0 \%$ \\
\hline & Tachy & 0 & 9 & 74 & $89.1 \%$ & $99.9 \%$ \\
\hline & PPV & $85.0 \%$ & $99.4 \%$ & $34.3 \%$ & & \\
\hline & NPV & $99.4 \%$ & $83.8 \%$ & $100 \%$ & & \\
\hline & $\mathbf{F}$ & $85.0 \%$ & $99.4 \%$ & $49.5 \%$ & & \\
\hline
\end{tabular}

Brady, bradycardia; Normal, normal HR; Tachy, tachycardia; $S e$, sensitivity; $S p$, specificity; $P P V$, positive predictive value; $N P V$, negative predictive value; $F$ : accuracy according to the F-score.

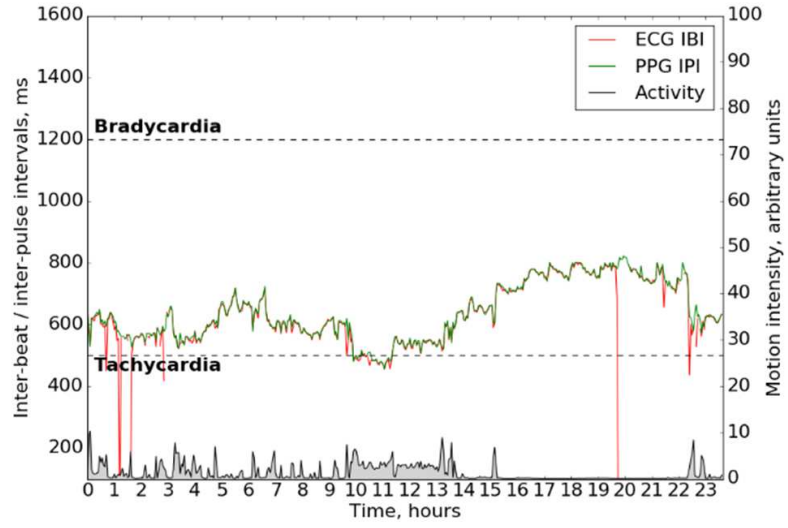

Figure 2. IPIs and IBIs recorded for a representative patient experiencing tachycardia during the 24-h monitoring. The bottom part of the figure also displays the movement intensity recorded with the accelerometer.

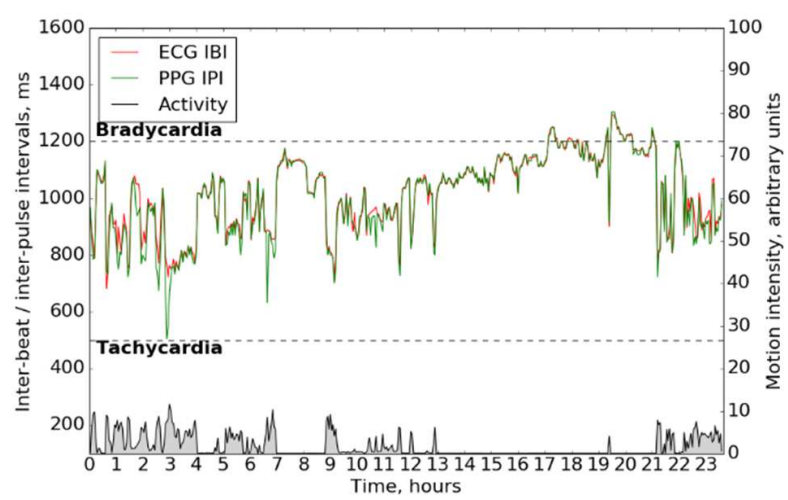

Figure 3. IPIs and IBIs recorded for a representative patient experiencing bradycardia during the 24-h monitoring. The bottom part of the figure also displays the movement intensity recorded with the accelerometer.

Examples of recorded IBI and IPI data for patients experiencing tachycardia and bradycardia are presented in Figures 2 and 3, respectively. Those figures also show at the bottom the movement intensity registered with the accelerometer during the free-living monitoring period.

Average IPI during bradycardia and tachycardia was $1310 \pm 80 \mathrm{~ms}$ and $459 \pm 37 \mathrm{~ms}$, respectively. IPI data could accurately detect episodes of bradycardia (Sensitivity: 85\%, Specificity: 99\%) and tachycardia (Sensitivity: 89\%, Specificity: 99\%). Normal rate detection showed 99\% Sensitivity and $85 \%$ Specificity (Table 1 ).

Accounting for movement intensity in the analysis reduced the number of available events of bradycardia and tachycardia. However, removing from the analysis those periods in which wrist movement were present improved the classification accuracy for tachycardia. A fair number of falsely detected events of tachycardia were reduced by removing IPIs in correspondence to high movement 
intensity (PPV from $24 \%$ to $34 \%$ ). At the same time, sensitivity for episodes of bradycardia increased (from $73 \%$ to $85 \%$ ) by accounting for movement intensity in the rejection of IPIs.

\section{Discussion}

This study shows that a wrist-wearable device equipped with PPG and accelerometer sensors can accurately detect HR abnormalities such as bradycardia and tachycardia in daily life in patients suffering from generic cardiac symptoms. The presented solution is particularly promising because it could offer continuous long-term monitoring capabilities at minimal burden for the patient and therefore considerably improve the way cardiac symptoms are investigated and documented.

Estimates of average HR from wrist PPG and acceleration data were valuable at the limits of physiological range to detect excessively fast and slow HR. However, we noted two substantial limitations: firstly, wrist movement of even low intensity tended to increase PPG-based estimates of HR which caused false detection of tachycardia and missed bradycardia events (Figure 4). Secondly, presence of frequent premature beats at rest could lead to underestimation of HR and thereby overestimation of IPIs as a result of missed PPG pulses. This caused erroneous adjudications of bradycardia while HR was still to be considered in a normal range (Figure 4).

Despite these limitations, our data shows a strong agreement between average IBIs and IPIs in free-living conditions irrespectively of wrist movement and freeliving activities. Furthermore, the reported limitations were substantially mitigated by the fact that only events longer than 3 minutes were considered as clinically relevant. Thus, the technical limitations of PPG may be overcome by the relatively slow transient nature of bradycardia and tachycardia in symptomatic patients.

\section{Conclusion}

A wrist-wearable device equipped with a PPG and acceleration sensor represents a promising, unobtrusive technology to detect HR abnormalities such as bradycardia and tachycardia in patients suffering from cardiac symptoms.

\section{Acknowledgements}

The authors would like to thank N. Sturkenboom (MD), L. Verborg (MD), R. Eerdekens (MD), L. van den Heuvel, and the personnel of the Holter department, and the Holter analysts of the Catharina hospital for their help and contributions to the data collection.
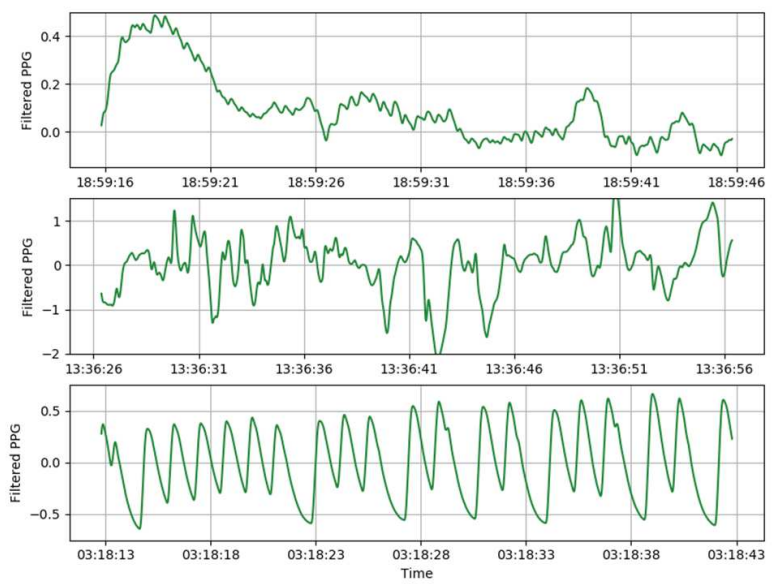

Figure 4. Raw PPG signal during: (top) tachycardia (mean IPI $=492 \mathrm{~ms}$ vs IBI $=460 \mathrm{~ms}$ ); (mid) false tachycardia due to motion artefacts (mean IPI $=469 \mathrm{~ms}$ vs IBI $=910 \mathrm{~ms}$ ); and (bottom) false bradycardia due to missed premature beats (mean IPI $=1580 \mathrm{~ms}$ vs IBI $=1110 \mathrm{~ms}$ ).

\section{References}

[1] Byrnes TJ, and Costantini O. Tachyarrhythmias and Bradyarrhythmias: Differential Diagnosis and Initial Management in the Primary Care Office. Medical Clinics of North America 2017; 101 (3):495-506.

[2] Page RL, Joglar JA, et al. 2015 ACC/AHA/HRS guideline for the management of adult patients with supraventricular tachycardia. Heart Rhythm 2016; 13:e136-221.

[3] Gupta S, Figueredo VM. Tachycardia mediated cardiomyopathy: pathophysiology, mechanisms, clinical features and management. Int J Cardiol 2014; 172:40-6.

[4] Rosero SP, Kutyifa V, Olshansky B, Zareb W. Ambulatory ECG monitoring in atrial fibrillation management. Prog in Cardiov Diseases 2013; 56:143-152.

[5] Gillinov S, Etiwy M, Wang R, Blackburn G, et al. Variable Accuracy of Wearable Heart Rate Monitors during Aerobic Exercise. Med Sci Sports Exerc 2017 Aug; 49(8):1697-1703.

[6] Bonomi AG, Schipper F, et al. Atrial Fibrillation Detection Using Photo-plethysmography and Acceleration Data at the Wrist. Computing in Cardiology 2016; 43.

[7] Bonomi AG, Goldenberg S, Papini G, Kraal J, et al. Predicting energy expenditure from photo-plethysmographic measurements of heart rate under beta blocker therapy: data driven personalization strategies based on mixed models. Conf Proc IEEE Eng Med Biol Soc 2015:7642-6.

[8] Valenti G, Westerterp KR. Optical heart rate monitoring module validation study. In IEEE International Conference on Consumer Electronics (ICCE) 2013; 195-196.

[9] van Andel J, Ungureanu C, Arends J, Aarts RM, et al. Using photoplethysmography in heart rate monitoring of patients with epilepsy. Epilepsy \& Behavior, 2015 45:142-145.

Address for correspondence:

Alberto G Bonomi.

HTC34, 5656AE, Eindhoven, the Netherlands.

alberto.bonomi@philips.com 